\title{
Absolute proper motion of the Galactic open cluster M $67^{\star}$
}

\author{
A. Bellini ${ }^{1,2, \star \star}$, L. R. Bedin ${ }^{2}$, B. Pichardo ${ }^{3}$, E. Moreno ${ }^{3}$, C. Allen ${ }^{3}$, G. Piotto ${ }^{1}$, and J. Anderson ${ }^{2}$ \\ 1 Dipartimento di Astronomia, Università di Padova, Vicolo dell'Osservatorio 3, 35122 Padova, Italy \\ e-mail: bellini@stsci.edu \\ 2 Space Telescope Science Institute, 3700 San Martin Drive, Baltimore, MD 21218, USA \\ 3 Instituto de Astronomía, Universidad Nacional Autónoma de México, Apartado Postal 70-264, 04510-México DF, Mexico
}

Received 15 December 2009 / Accepted 23 February 2010

\section{ABSTRACT}

\begin{abstract}
We derived the absolute proper motion (PM) of the old, solar-metallicity Galactic open cluster M 67 using observations collected with CFHT (1997) and with LBT (2007). About 50 galaxies with relatively sharp nuclei allow us to determine the absolute PM of the cluster. We find $\left(\mu_{\alpha} \cos \delta, \mu_{\delta}\right)_{\mathrm{J} 2000.0}=(-9.6 \pm 1.1,-3.7 \pm 0.8)$ mas $\mathrm{yr}^{-1}$. By adopting a line-of-sight velocity of $33.78 \pm 0.18 \mathrm{~km} \mathrm{~s}^{-1}$, and assuming a distance of $815 \pm 50 \mathrm{pc}$, we explore the influence of the Galactic potential, with and without the bar and/or spiral arms, on the galactic orbit of the cluster.
\end{abstract}

Key words. astrometry - open cluster and associations: NGC 2682 (M 67) - Galaxy: kinematics and dynamics

\section{Introduction}

The solar-metallicity Galactic open cluster M 67 (NGC 2682) is among the most-studied Galactic open clusters. Still, its absolute proper motion (PM) remains poorly constrained.

We applied for the first time on ground-based multiepoch CCD wide-field images the PM techniques developed in Anderson et al. (2006, Paper I), Yadav et al. (2008, Paper II), Bellini et al. (2009, Paper III) to define an absolute reference frame using background faint galaxies. In the next section we describe the data set and the measurements, and a final section is dedicated to the study of the orbit of M67 within the Galaxy under different assumptions for the Galactic potential.

\section{Observations, data reduction, proper motions}

Two data sets - collected with two different telescopes and at two different epochs - were used to measure the PM of objects in the field of M 67.

As the first epoch (hereafter, epoch 1) we employed images taken on Jan. 10-13, 1997, at the CFHT 3.6m telescope. These

* Based on data acquired with the Large Binocular Telescope (LBT) at Mt. Graham, Arizona, under the Commissioning of the Large Binocular Blue Camera. The LBT is an international collaboration among institutions in the United States, Italy and Germany. LBT Corporation partners are the University of Arizona on behalf of the Arizona university system; Istituto Nazionale di Astrofisica, Italy; LBT Beteiligungsgesellschaft, Germany, representing the MaxPlanck Society, the Astrophysical Institute Potsdam, and Heidelberg University; the Ohio State University, and the Research Corporation, on behalf of the University of Notre Dame, University of Minnesota and University of Virginia; and on observations obtained at the CanadaFrance-Hawaii Telescope (CFHT), which is operated by the National Research Council of Canada, the Institut National des Sciences de l'Univers of the Centre National de la Recherche Scientifique of France, and the University of Hawaii.

$\star \star$ Visiting PhD Student at STScI under the "2008 graduate research assistantship" program. images were first presented by Richer et al. (1998). We took only a subsample of this data set, specifically 15 exposures of $1200 \mathrm{~s}$ in the $V$ filter, with a median value for seeing and airmass of 1 " and 1.2 respectively. Each image was collected with the UH8K camera ( $8 \mathrm{CCDs}, 2 \mathrm{~K} \times 4 \mathrm{~K}$ pixels each, with an average scale of 210 mas pixel $^{-1}$ ) covering a field-of-view $(\mathrm{FoV})$ of $\sim 29^{\prime} \times 29^{\prime}$.

The second-epoch (epoch 2) data were collected between Feb. 16 and Mar. 182007 and consist of 56 images of $180 \mathrm{~s}$ exposures in the $B$-band filter and 42 exposures of 100-110 s in the $V$-band, obtained with the LBC-blue camera (4 CCDs, $2 \mathrm{~K} \times 4.5 \mathrm{~K}$ pixels each, FoV of $\left.\sim 24^{\prime} \times 26^{\prime}\right)$. A large dither pattern $(\sim 30 \%$ of the FoV) was employed for both filters. Median seeing and airmass were $1^{\prime \prime}$ and 1.1 for the $V$, and $1 . .3$ and 1.1 for the $B$ images. We selected only $B$ images for finding objects, while those in $V$ were taken to provide all the astrometric information. A more extensive description of the LBT data set is given in three companion papers (Bellini \& Bedin 2010; Bellini et al. 2010; and Bellini et al. in prep.). Procedures and algorithms used to derive the list of objects, star positions and fluxes, and PMs are those explained in great detail in Paper I and in Anderson et al. (2008, A08). Below we briefly describe these reduction procedures, which were organized in three steps.

In the first step, we employed the software described in Paper I to obtain PSFs, star positions and fluxes in each chip of each exposure and for the best sources (bright, isolated, with a stellar profile). We corrected LBC raw positions for geometric distortion (GD), as described in Bellini \& Bedin (2010), and we used these corrected positions to register all of the LBC singlechip images into a common distortion-free reference frame (the master frame) by means of linear transformations. With this master frame we derived the GD correction also for the UH8K positions, using the same technique (used also in Bellini \& Bedin 2009).

The second step consisted of producing the list of objects (the target list). We took every single local maximum detected within each individual chip of each LBC $B$ image to build a peak-map image (A08). The peak map consists of an image with 
the same pixel scale of the master frame, where we added 1 to a pixel count each time a local maximum, measured in a given image, fell within that pixel (once transformed with the aforementioned linear transformations). A $3 \times 3$ box-car filter is applied to the peak map, and in our final list we considered as significant any object $3.2 \sigma$ (where $\sigma$ is the rms of the peak-map background) above the local surrounding. Our list contains objects generating a local maximum in at least $60 \%$ of the images covering the patch of sky where the maximum is found. We additionally required a minimum coverage of $10 \mathrm{~B}$ images.

With the $B$ images we generated the target list because they are more numerous and have a lower background compared to $V$ images. In addition, large dithers helped us to produce a list of solid detections even for the faintest sources. We further purged our list from excessively faint sources, keeping only objects with at least $100 \mathrm{DN}$ above the local background and which showed up in at least one observation for each of the two $V$-filter epochs. We also excluded objects within chip \# 2 and 4 of the UH8K camera, because these chips are highly affected by charge transfer inefficiency. As a consequence, the useful FoV is reduced by $25 \%$.

In the last step we derived PMs (see Sect. 7 of Paper I for more details): We measured each object in the target list in each chip of each $V$ exposure where it could be found, using PSFfitting to get a chip-based flux and a GD-corrected position. Then we organized the images in pairs of one image from the first and one from the second epoch. For each object in each pair we computed the displacement (in the reference frame) between where epoch 1 predicts the object position in epoch 2 , and the actually-observed position in epoch 2. Multiple measurements of displacements for the same object were then used to compute average displacements and rms.

It is clear that to make these predictions, we needed a set of objects as a reference to compute positional transformations between the two epochs for each source. The cluster members of M 67 were a natural choice, as their internal motion is within our

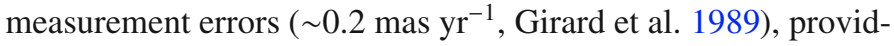
ing an almost rigid reference system with the common systemic motion of the cluster.

We initially identified cluster members according to their location in the $V$ vs. $B-V$ color-magnitude diagram. We took only main sequence (MS) stars to transform each exposure into the master frame, because it spans a narrow range in color ${ }^{1}$. By predominantly using cluster members, we ensured the PMs to be measured relative to the bulk motion of the cluster. We iteratively removed from the member list those objects with a field-type motion (i.e., with a PM larger than 2.3 mas $\mathrm{yr}^{-1}$ from the mean M 67 motion), even though their colors may have placed them near the cluster MS. This particular cut value in the PMs represents a compromise between loosing (poorly measured) M 67 members and including field-type objects. Our final member list contains 209 color- and PM-selected objects. In order to minimize the influence of any uncorrected GD residual, PMs for each object were computed with a local sample of members; specifically the 25 (at least) closest $\left(r<3^{\prime}\right)$, well-measured cluster stars (see Paper I for more details).

\footnotetext{
1 Note that it was not possible to use the high-probability members derived in Paper II as starting reference members, because there are not enough stars in common between the two catalogs. Indeed, the PM catalog of Paper II includes stars in the magnitude range $9<V<21$ (with reliable PM measurements down to $V \sim 19$ ), while our new PM determinations are in the magnitude range $18<V<26$.
}

Then we matched our master frame with the sources in the Digital Sky Survey (DSS) to compute scale and orientation for our master frame. We needed to know scale and orientation with an error of $\sim 1 \%$. Even if only saturated stars could be matched with the DSS catalog, the used sample was good enough for this purpose. We divided our displacements for the time baseline between the two epochs (10.13 years) to obtain PMs in units of mas $\mathrm{yr}^{-1}$. We kept in our final PM list only objects with at least two measurements of the displacement, and with PM rms $<7 \mathrm{mas} \mathrm{yr}^{-1}$ in each coordinate. Finally, we corrected our PMs for differential chromatic refraction (DCR) effects, as done in Papers I and III, using M 67 white dwarfs and MS stars. We note

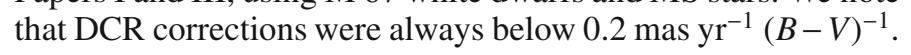

On the basis of the PM dispersion of members $\left(\sim 0.9\right.$ mas $\left.\mathrm{yr}^{-1}\right)$ with respect to their mean (which reflects our measurement errors) we estimated the uncertainty on the adopted member reference system to be $\pm(0.1,0.1)$ mas $\mathrm{yr}^{-1}$. (Note that our estimate of the member dispersion is somehow biased because of the 2.3 mas $\mathrm{yr}^{-1}$ membership selection criterion we adopted in our proper-motion determination. Hence, the dispersion could be underestimated. Nevertheless, it still provides a good indication of the involved errors.) We were still left with the problem of finding the zero point of our PMs which for now were only relative to the cluster's mean motion and not to an absolute reference system. Unfortunately, it was not possible to directly link our proper motions to the UCAC ${ }^{2}$ catalog (Zacharias et al. 2009), which includes stars mainly in the 8 to 16 magnitude range in a single bandpass between $V$ and $R$, with no overlap with our catalog.

Background galaxies can be considered as fixed points in the sky, and provide an excellent, and directly-observable absolute reference system. A visual inspection of the images revealed many such galaxies. About 100 of them show point-like nuclei, which could be fitted with our PSFs to measure positions and PMs (as done in Bedin et al. 2003, 2006).

As expected, the errors of galaxy positions are several times larger than the typical error of star positions, and depend strongly on galaxy morphology. The comparison of the galaxy PMs (which should all be the same), suggests that we are underestimating their errors (which is not the case, for the point sources), as a result of a complex combination of seeing and galaxy shape. For this reason, we did not use their weighted mean to estimate the centroid of the galaxy PMs, but adopted an iterative $\sigma_{\mu}$-clipped average instead.

We started with all the $N^{\mathrm{G}} \simeq 100$ galaxies in the sample, for which the motion is within 20 mas $^{-1}{ }^{-1}$ from the first-guess mean PM of galaxies, and we iteratively estimated their PM dispersion $\sigma_{\mu}^{\mathrm{G}}$ as the 1-D 68.27th-percentile of their distribution around the median motion. We then excluded from the sample those galaxies with $\mathrm{PM}>2.5 \sigma_{\mu}^{\mathrm{G}}$ from the median galaxies' $\mathrm{PM}$, and we rederived new values of $\sigma_{\mu}^{\mathrm{G}}$, median motion and $N^{\mathrm{G}}$. We iterated this procedure 10 times, noting that the values of median, $\sigma_{\mu}^{\mathrm{G}}$, and $N^{\mathrm{G}}$ converged after eight iterations. Final values for $\sigma_{\mu}^{\mathrm{G}}$ and $N^{\mathrm{G}}$ are 2.3 mas $\mathrm{yr}^{-1}$ and 47 , respectively.

By adopting as the origin of the absolute PM system the final median value of the PM of galaxies, we found for M 67 an absolute PM (J2000.0) of

$\left(\mu_{\alpha} \cos \delta, \mu_{\delta}\right)=(-9.6,-3.7) \pm(1.1,0.8) \mathrm{mas} \mathrm{yr}^{-1}$

\footnotetext{
2 http://www . usno.navy .mil/USNO/astrometry/ optical-IR-prod/ucac/.
} 


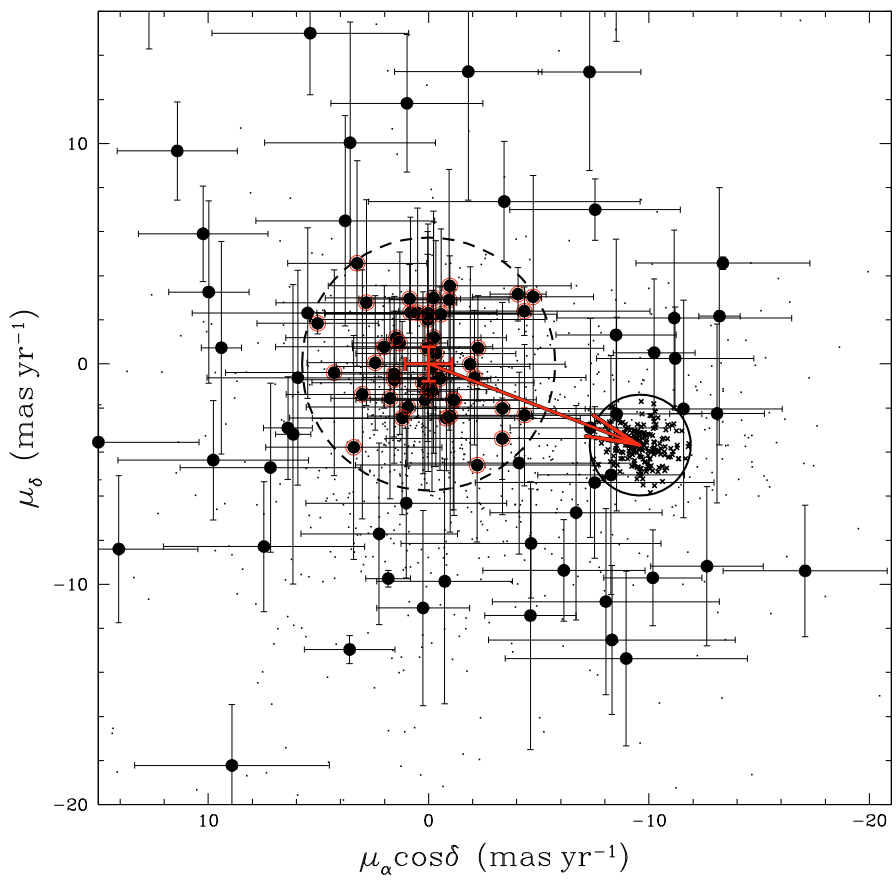

Fig. 1. Vector-point diagram of the absolute PMs, in equatorial coordinates (J2000.0). Small crosses are M 67 members (within the solid circle), filled circles are the reference galaxies, 47 of which (marked with red open circles) were taken to compute the centroid of the galaxy distribution.

where the uncertainties come from adding in quadrature the uncertainties of the centroid of M 67 members to the error of the location of the centroid of the galaxies (for each coordinate independently).

The entire sample of $\sim 100$ galaxies was used to estimate the uncertainty associated with the displacement of a single, typical galaxy. This was calculated as the 1-D 68.27th-percentile of the distribution of the galaxies around their median displacement (as

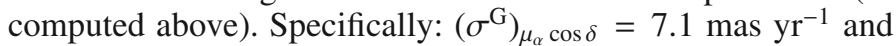
$\left(\sigma^{\mathrm{G}}\right)_{\mu_{\delta}}=5.3$ mas $\mathrm{yr}^{-1}$. Because we only used the most consistent subset of 47 galaxies to derive their relative median motion, we associated to the median of their displacements an uncertainty that statistically takes into account only those $N^{\mathrm{G}}=47$ galaxies. This is done by reducing the error on the single average galaxy by the factor $1 / \sqrt{N^{\mathrm{G}}-1}$. This more-conservative approach might result in a overestimate of our internal errors. However, considering all the uncertainties involved in the assignment of an error to a galaxy displacement and their limited number, this is a preferable approach.

Figure 1 shows our absolute vector-point diagram (VPD) for all the objects in the final list (small dots). M67 members are marked with small crosses. These are the stars within

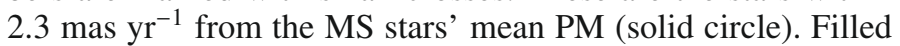
circles are visually-confirmed galaxies, and are shown with our estimated error bars. The best galaxies (selected with the aforementioned iterative procedure) are highlighted with red open cir-

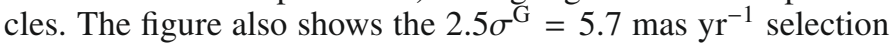
radius (dashed circle). The red arrow indicates our estimate of the absolute PM of M 67. The error bars at the base of the arrow indicate the total uncertainty (dominated largely by the estimates in the centroid of the relative PM distribution of the background galaxies). In Fig. 2, we used the same symbols as in Fig. 1 to show that there are no clear systematic errors in the galaxy PMs
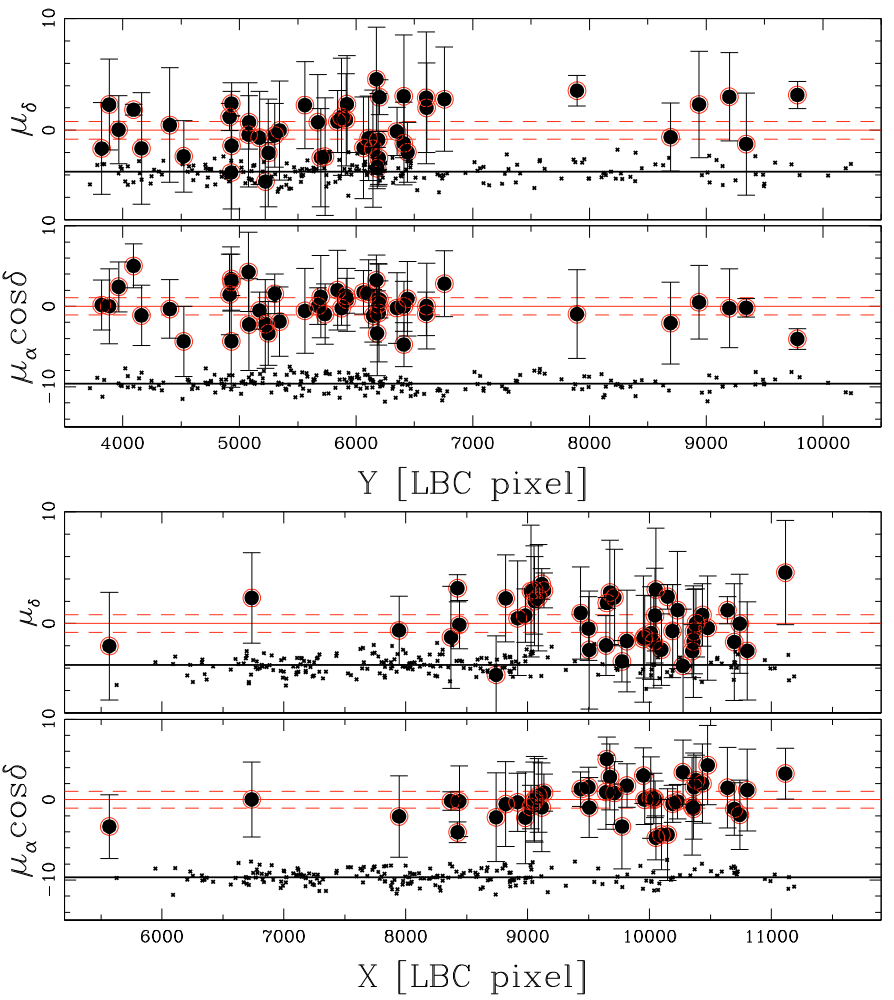

Fig. 2. From top to bottom, $\mu_{\delta}$ and $\mu_{\alpha} \cos \delta$ as a function of $X$ and $Y$. When PMs are plotted as a function of their position in the master frame $(X, Y)$, no clear systematic errors appear. Note that two chips of UH8K are not used $(Y>7000$, for $X<5500$ and $X>9500)$, and that the cluster center is at location $(X, Y) \simeq(7500,7000)$. These two effects both create gaps in the galaxy spatial distribution.

as a function of the coordinates of the master frame $(X$, and $Y$, parallel to $\alpha$ and $\delta$, respectively).

The absolute PM of M 67 has been measured by several authors. The first of these determinations comes from van Rhijn (1922), with $\left(\mu_{\alpha} \cos \delta, \mu_{\delta}\right)=(-7,+2) \pm(9,9) \mathrm{mas} \mathrm{yr}^{-1}$. Many years later, Murray (1968) computed $\left(\mu_{\alpha} \cos \delta, \mu_{\delta}\right)=$ $(-9.4,-7.0) \pm(8.0,2.3)$ mas $\mathrm{yr}^{-1}$. The more contemporary work of Baumgardt et al. (2000), which made use of the two faint Hipparcos stars HIP-43491, HIP-43465 $(V \sim 10)$, reports: $\left(\mu_{\alpha} \cos \delta, \mu_{\delta}\right)=(-6.47,-6.27) \pm(1.29,1.01) \mathrm{mas} \mathrm{yr}^{-1}$. Finally, Kharchenko et al. (2005) selected 27 M 67 members on the basis of their absolute PMs, as derived from the ASCC-2.5 catalog (Kharchenko 2001) - which is based on HipparcosTycho family catalogs - and on their location on the $V$ vs. $B-V$ color-magnitude diagram. The obtained mean-absolute motion of these 27 members is $\left(\mu_{\alpha} \cos \delta, \mu_{\delta}\right)=(-8.31,-4.81) \pm$ $(0.26,0.22){\text { mas } y r^{-1}}$.

Figure 3 shows (in blue) three stars in the field with Hipparcos PMs (HIP-43491, HIP-43465 and HIP-43519, with error bars), and the stars from the Tycho-2 catalog (in green). In the same plot, we mark with a red arrow our derived M 67 absolute PM, where the estimated PM error is indicated with an ellipse. Note that both the Hipparcos and the Tycho-2 stars are far too bright to be measured in our survey. Our M 67 absolute PM determination is marginally consistent with the bulk of Tycho- 2 measurements for the objects in the same field. This is in line with the expected accuracies for Tycho-2. The same figure also shows (dashed arrows) previous determinations of the M 67 absolute motion. 
Table 1. Non-axisymmetric Galactic model (Pichardo et al. 2003, 2004).

\begin{tabular}{lcl}
\hline \hline Parameter & Value & References \\
\hline Bar half-length & $3.1-3.5 \mathrm{kpc}$ & Gerhard (2002) \\
Bar axial ratios & $10: 3.8: 2.6$ & Freudenreich (1998) \\
Bar scale lengths & $1.7,0.64,0.44 \mathrm{kpc}$ & Freudenreich (1998) \\
Bar angle (respect to the Sun) & $20^{\circ}$ & Gerhard (2002) \\
Bar mass & $10^{10} M_{\odot}$ & Debattista et al. (2002) \\
Bar pattern speed $\left(\Omega_{\mathrm{B}}\right)$ & $30-60 \mathrm{~km} \mathrm{~s}^{-1} \mathrm{kpc}^{-1}$ & $\left(^{*}\right)$ \\
Spiral Arms locus & Bisymmetric $($ Logthm) & Churchwell et al. (2009) \\
Spiral Arms pitch angle & $15.5^{\circ}$ & Drimmel (2000) \\
Spiral Arms external limit & $12 \mathrm{kpc}$ & Drimmel (2000) \\
Spiral Arms: exp. with scale-length & $2.5 \mathrm{kpc}$ & Disk based \\
Spiral Arms force contrast & $\sim 10 \%$ & Patsis et al. (1991) \\
Spiral Arms pattern speed $\left(\Omega_{\mathrm{S}}\right)$ & $20 \mathrm{~km} \mathrm{~s}^{-1} \mathrm{kpc}^{-1}$ & Martos et al. (2004) \\
\hline
\end{tabular}

References. ${ }^{*}$ ) Weiner \& Sellwood (1999), Fux (1999), Ibata \& Gilmore (1995), Englmaier \& Gerhard (1999).

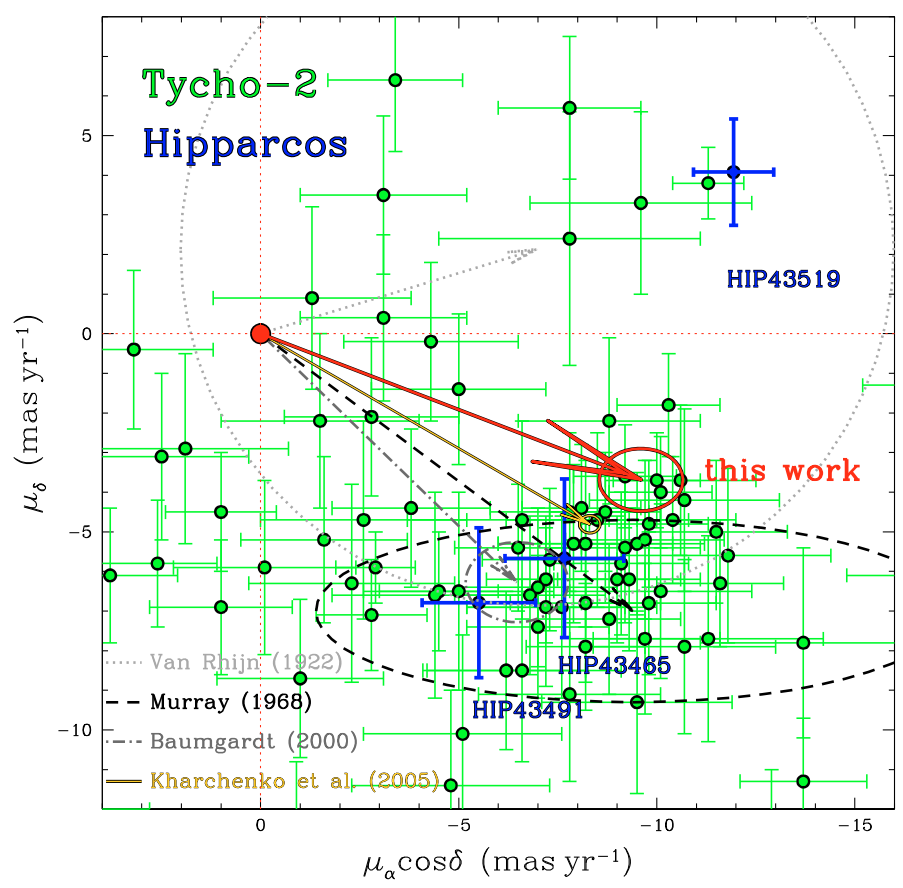

Fig. 3. Comparison of our derived absolute PMs with values from the literature. The three blue points are Hipparcos PM determinations, while green points are from the Tycho-2 catalog. Our M 67 absolute $\mathrm{PM}$ is indicated with a red arrow, and the ellipse in its tip shows our uncertainty. The other four arrows in different colors and types refer to previous values.

We emphasize that the absolute PM presented here is based on direct observations of background galaxies, used to define the absolute reference frame. It is a purely differential measurement, which does not rely, as do previous measurements, on a complex registration to the International Celestial Reference System through a global network of objects. We end this section noticing the good agreement between the absolute PM value of M 67 as derived with the bright sources of the Hipparcos cata$\log$ (Kharchenko et al. 2005) and that based on the faint, "fix" galaxies (this work).

\section{The Galactic orbit of M 67}

With the absolute PM of M 67 given in the previous section, its line-of-sight velocity of $33.78 \pm 0.18 \mathrm{~km} \mathrm{~s}^{-1}$ and its heliocentric distance of $815 \pm 50 \mathrm{pc}$ (both from Paper II), we have computed the Galactic orbit of M 67. We employed a Galactic potential that includes the axisymmetric model of Allen \& Santillán (1991) and the bar and spiral arms models of Pichardo et al. $(2003,2004)$. The axisymmetric background potential of Allen \& Santillán (1991) has been scaled to give a rotation velocity of $254 \mathrm{~km} \mathrm{~s}^{-1}$ at the solar position, based on the most-recent radio astrometry observations by Reid et al. (2009). We keep the original value $R_{0}=8.5 \mathrm{kpc}$ of the solar galactocentric distance (Reid et al. (2009) give $R_{0}=8.4 \pm 0.6 \mathrm{kpc}$ ). The adopted parameters for the bar and spiral arms and the corresponding references are provided in Table 1 . The values of the parameters are based on recent observations of the Milky Way. The bar model is an inhomogeneous ellipsoidal potential that closely approximates model S of Freudenreich (1998) from the COBE/DIRBE data of the Galactic bar. For the spiral perturbation, Pichardo et al. (2003) refined their model until self-consistent orbital solutions were found. The orbital self-consistency of the spiral arms was tested through the reinforcement of the spiral potential by the stellar orbits (Patsis et al. 1991). For an extensive description of the models, see Pichardo et al. (2003, 2004).

Table 2 gives the local standard of rest (LSR) initial velocity $(U, V, W)$ and the corresponding cylindrical components $(\Pi, \Theta)$ for three different heliocentric distances of M 67 (central, minimum, and maximum). We used the solar motion $(U, V, W)=$ $(-10,5.2,7.2) \mathrm{km} \mathrm{s}^{-1}$ (Dehnen \& Binney 1998). $U$ is negative toward the Galactic center. In Table 3 we give some parameters of the Galactic orbits, corresponding to the three heliocentric distances, computed backwards in time during $1 \mathrm{Gyr}$ in the scaled axisymmetric potential. Columns 2 and 3 show the minimum and maximum galactocentric distances and Col. 4 the maximum $z$-distance from the Galactic plane; the orbital eccentricity is given in Col. 5; Cols. 6 to 8 give the azimuthal, radial, and vertical periods respectively, and the last two columns the $z-$ component of the angular momentum and energy per unit mass.

Previous computations of the Galactic orbit of M 67 were made by Keenan et al. (1973) and Allen \& Martos (1988) in other axisymmetric potentials. Keenan et al. (1973) used the Galactic models of Schmidt (1956) (with an $R_{0}$ value of $8.2 \mathrm{kpc}$ and a circular velocity at the Sun's position $V_{0}=216 \mathrm{~km} \mathrm{~s}^{-1}$ ) and of Innanen (1966) (with an $R_{0}=10 \mathrm{kpc}$ and a $V_{0}=$ $250 \mathrm{~km} \mathrm{~s}^{-1}$ ). They obtained $R_{\max }=8.8 \mathrm{kpc}$ and $10.7 \mathrm{kpc}$, respectively, $z_{\max }=0.4 \mathrm{kpc}$, and an eccentricity of 0.1 for both models. Allen \& Martos (1988) used their galactic model (Allen \& Martos 1986) with $R_{0}=8 \mathrm{kpc}$ and $V_{0}=225 \mathrm{~km} \mathrm{~s}^{-1}$. They obtained $R_{\max }=8.7 \mathrm{kpc}, z_{\max }=0.48 \mathrm{kpc}$ and an eccentricity of 0.11 . In all cases, the orbit of M 67 has a small eccentricity, 
A. Bellini et al.: Absolute proper motion of the Galactic open cluster M 67

Table 2. Local standard of rest initial velocity $(U, V, W)$ and Galactic $(\Pi, \Theta)\left(\mathrm{km} \mathrm{s}^{-1}\right)$ for the three heliocentric distances $d(\mathrm{kpc})$.

\begin{tabular}{cccccc}
\hline \hline$d$ & $U$ & $V$ & $W$ & $\Pi$ & $\Theta$ \\
\hline 0.815 & $31.92 \pm 3.4$ & $-21.66 \pm 3.7$ & $-8.71 \pm 4.3$ & $21.55 \pm 3.2$ & $233.53 \pm 3.8$ \\
0.765 & $30.77 \pm 3.0$ & $-21.03 \pm 3.4$ & $-6.64 \pm 3.6$ & $20.98 \pm 3.0$ & $234.05 \pm 3.5$ \\
0.865 & $33.06 \pm 3.4$ & $-22.28 \pm 3.9$ & $-10.78 \pm 4.1$ & $22.12 \pm 3.4$ & $233.02 \pm 4.0$ \\
\hline
\end{tabular}

Table 3. Orbit parameters in the scaled axisymmetric potential for the three heliocentric distances.

\begin{tabular}{cccccccccc}
\hline \hline $\begin{array}{c}d \\
(\mathrm{kpc})\end{array}$ & $\begin{array}{c}r_{\min } \\
(\mathrm{kpc})\end{array}$ & $\begin{array}{c}r_{\max } \\
(\mathrm{kpc})\end{array}$ & $\begin{array}{c}z_{\max } \\
(\mathrm{kpc})\end{array}$ & $e$ & $\begin{array}{c}P_{\phi} \\
(\mathrm{Myr})\end{array}$ & $\begin{array}{c}P_{r} \\
(\mathrm{Myr})\end{array}$ & $\begin{array}{c}P_{z} \\
(\mathrm{Myr})\end{array}$ & $\begin{array}{c}h \\
\left(\mathrm{kpc} \mathrm{km} \mathrm{s}^{-1}\right)\end{array}$ & $\begin{array}{c}E \\
\left(10^{2} \mathrm{~km}^{2} \mathrm{~s}^{-2}\right)\end{array}$ \\
\hline 0.815 & 7.65 & 9.28 & 0.46 & 0.096 & 207 & 146 & 76 & 2118.3 & -1589.38 \\
0.765 & 7.65 & 9.24 & 0.42 & 0.094 & 206 & 145 & 74 & 2114.7 & -1591.50 \\
0.865 & 7.66 & 9.33 & 0.50 & 0.098 & 208 & 147 & 78 & 2122.0 & -1587.21 \\
\hline
\end{tabular}

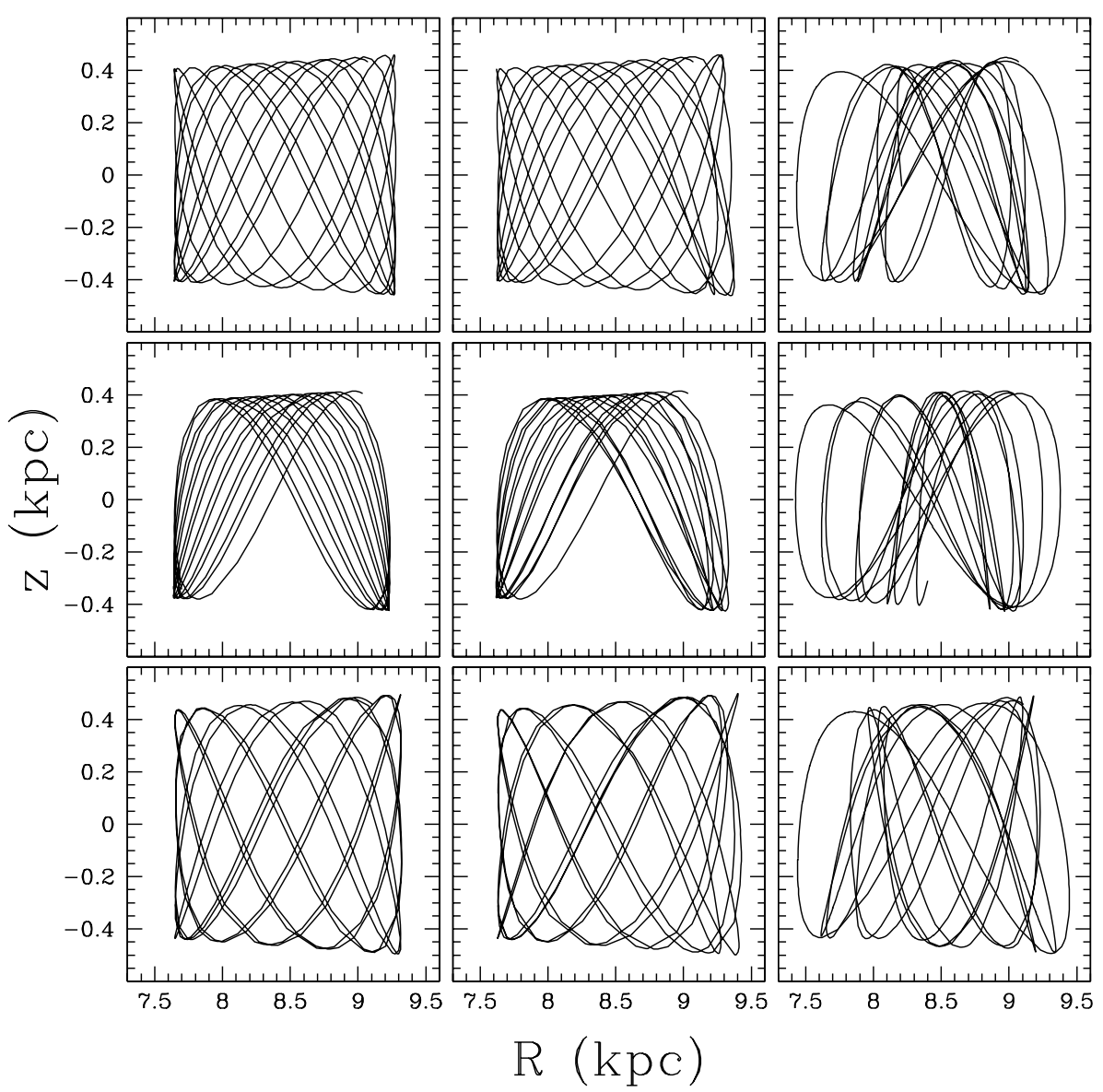

Fig. 4. Meridional orbits using the three heliocentric distances; $d=0.815 \mathrm{kpc}$ (upper panels), $0.765 \mathrm{kpc}$ (middle panels), and $0.865 \mathrm{kpc}$ (lower panels), computed in the axisymmetric (left panels), barred (central panels), and with bar + spiral arms (right panels) scaled Galactic potential. and the differences found are mostly attributable to the different Galactic parameters and values for the solar motion employed. Indeed, the different values obtained for $R_{\max }$ result mostly from the scaling of the Sun-center distance, as shown by the ratios $R_{0} / R_{\max }$, which are all between 1.07 and 1.09 . The more contemporary, much-improved Galactic models and the precise value now available for the absolute proper motion of M 67 should result in a more reliable orbit for this cluster.

We also computed the Galactic orbit of M 67 in the nonaxisymmetric potential, first including only the bar, then with the bar + spiral arms. The scaled background axisymmetric potential was considered. The angular velocity of the bar was taken as $\Omega_{B}=60 \mathrm{~km} \mathrm{~s}^{-1} \mathrm{kpc}^{-1}$; the other parameters of the nonaxisymmetric components are given in Table 1 . In the case with spiral arms, the mass of these arms was taken as $2.2 \%$ the mass of the scaled disk component, which is $3 \%$ of the mass of the original disk. This mass gives a force contrast as listed in Table 1.
Figure 4 shows the meridional orbits computed in the axisymmetric potential (left panels), axisymmetric + bar potential (central panels) and axisymmetric + bar + spiral arms potential (right panels), using the three heliocentric distances. As shown, the potential that includes only the Galactic bar gives an orbit similar to that obtained with the axisymmetric potential. This is because the orbit of M 67 lies far outside the region of the bar. However, the potential that includes both the bar and spiral arms shows a different behavior. The orbit is perturbed by the spiral arms, mainly in the radial direction. The radial dispersion is not very strong in M 67, but distorts what would be a box orbit. Thus, a moderate spiral potential has important effects in the kinematics of orbits near the Galactic plane, as is the case in M 67, and in general, as is the case for the solar-neighborhood stars (Antoja et al. 2009). This result holds for the allowed variations in proper motion, radial velocity and distance, because the most important parameter that affects the orbit is the mass of the spiral arms. 

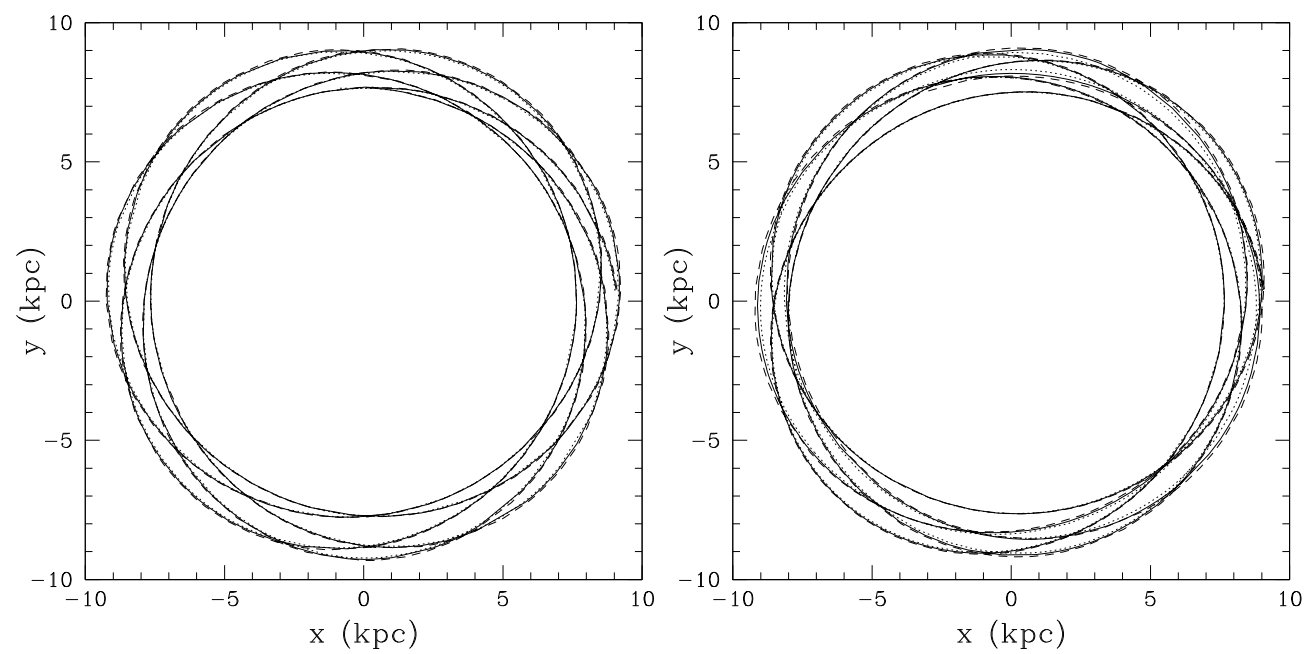

Fig. 5. Projection of the orbit on the Galactic plane computed in the scaled axisymmetric potential (left), and in the bar + spiral arms scaled Galactic potential (right). We adopted an heliocentric distance $d=0.815 \mathrm{kpc}$ (full line), $0.765 \mathrm{kpc}$ (dotted line), and $0.865 \mathrm{kpc}$ (dashed line).

In left panel of Fig. 5 we show the projection of the orbit on the Galactic plane, computed in the axisymmetric potential. At the scale shown in this figure there is no appreciable difference between the orbits using the three heliocentric distances. The right panel shows the corresponding orbits in the axisymmetric + bar + spiral arms potential. There is a slight difference in the azimuthal behavior in both figures.

We have also computed the tidal radius of M 67 in the axisymmetric + bar + spiral arms scaled potential. With a total mass in M 67 of $279 M_{\odot}$, listed by Piskunov et al. (2008), using King's equation (King 1962) we obtained a mean tidal radius of $7.1 \mathrm{pc}$. With the alternative Eq. (1) in Allen et al. (2006) the tidal radius is $8 \mathrm{pc}$. Both results are near the $9.6 \mathrm{pc}$ value listed by Piskunov et al. (2008).

Acknowledgements. We thank the anonymous referee for a careful reading of the manuscript and useful comments. A.B. acknowledges the support by the CA.RI.PA.RO. foundation, and by the STScI under the "2008 graduate research assistantship" program. B.P. thanks project UNAM through grant PAPIIT IN1 19708. G.P. acknowledges partial support by PRIN07 (prot. 20075TP5K9) and by ASI under the program ASI-INAF I/016/07/0 program.

\section{References}

Allen, C., \& Martos, M. A. 1986, Rev. Mex. Astron. Astrofis., 13, 137 Allen, C., \& Martos, M. A. 1988, Rev. Mex. Astron. Astrofis., 16, 25 Allen, C., \& Santillán, A . 1991, Rev. Mex. Astron. Astrofis., 22, 255 Allen, C., Moreno, E., \& Pichardo, B. 2006, ApJ, 652, 1150

Antoja, T., Valenzuela, O., Pichardo, B., et al. 2009, ApJ, 700, 78

Anderson, J., , Bedin, L. R., Piotto, G., Yadav, R. S., \& Bellini, A. 2006, A\&A, 454, 1029 (Paper I)

Anderson, J., Sarajedini, A., Bedin, L. R., et al. 2008, AJ, 135, 2055 (A08)

Baumgardt, H., Dettbarn, C., \& Wielen, R. 2000, A\&AS, 146, 251

Bedin, L. R., Piotto, G., King, I. R., \& Anderson, J. 2003, AJ, 126, 247
Bedin, L. R., Piotto, G., Carraro, G., King, I. R., \& Anderson, J. 2006, A\&A, 460, L27

Bellini, A., \& Bedin, L. R. 2009, PASP, 121, 1419

Bellini, A., \& Bedin, L. R. 2010, A\&A, submitted

Bellini, A., Piotto, G., Bedin, L. R., et al. 2009, A\&A, 493, 959 (Paper III)

Bellini, A., Bedin, L. R., Piotto, G., et al. 2010, A\&A, 513, A50

Churchwell, E., Babler, B. L., Meade, M. R., et al. 2009, PASP, 121, 213

Debattista, V. P., Gerhard, O., \& Sevenster, M. N. 2002, MNRAS, 334, 355

Dehnen, W., \& Binney, J. 1998, MNRAS, 298, 387

Drimmel, R. 2000, A\&A, 358, 13

Englmaier, P., \& Gerhard, O. 1999, MNRAS, 304, 512

Freudenreich, H. T. 1998, ApJ, 492, 495

Fux, R. 1999, A\&A, 345, 787

Gerhard, O. 2002, ASPC, 273, 73

Girard, T. M., Grundy, W. M., Lopez, C. E., \& van Altena, W. F. 1989, AJ, 98, 227

Ibata, R. A., \& Gilmore, G. F. 1995, MNRAS, 275, 605

Innanen, K. A. 1966, ApJ, 143, 153

Keenan, D. W., Innanen, K. A., \& House, F. C. 1973, AJ, 78, 173

King, I. R. 1962, AJ, 67, 471

Kharchenko, N. V. 2001, Kinematika i Fizika Nebesnykh Tel, 17, 409

Kharchenko, N. V., Piskunov, A. E., Röser, S., Schilbach, E., \& Scholz, R.-D. 2005, A\&A, 438, 1163

Martos, M., Hernández, X., Yáñez, M., Moreno, E., \& Pichardo, B. 2004, MNRAS, 350, 47

Murray, C. A. 1968, Royal Greenwich Observatory Bulletin, 141, 339

Patsis, P. A., Contopoulos, G., \& Grosbol, P. 1991, A\&A, 243, 373

Pichardo, B., Martos, M., Moreno, E., \& Espresate, J. 2003, ApJ, 582, 230

Pichardo, B., Martos, M., \& Moreno, E. 2004, ApJ, 609, 144

Piskunov, A. E., Schilbach, E., Kharchenko, N. V., Röser, S., \& Scholz R. -D. 2008, A\&A, 477, 165

Reid, M. J., Menten, K. M., Zheng, X. W., et al. 2009, ApJ, 700, 137

Richer, H. B., Fahlman, G. G., Rosvick, J., \& Ibata, R. 1998, ApJ, 504, L91

Schmidt, M. 1956, BAN, 13, 15

van Rhijn, P. J. 1922, Pub. of the Kapteyn Astronomical Lab. Groningen, 33, 1

Weiner, B. J., \& Sellwood, J. A. 1999, ApJ, 524, 112

Yadav, R. K. S., Bedin, L. R., Piotto, G., et al. 2008, A\&A, 484, 609 (Paper II) Zacharias, N., Finch, C., Girard, T., et al. 2009, VizieR Online Data Catalog, 1315 